\title{
МАТЕМАТИЧЕСКАЯ МОДЕЛЬ ИНЖЕКЦИИ СЕРОВОДОРОДА В ПЛАСТ, ЧАСТИЧНО НАСЫЩЕННЫЙ ВОДОЙ
}

\author{
Хасанов Марат Камилович, \\ hasanovmk@mail.ru \\ Стерлитамакский филиал Башкирского государственного университета, \\ Россия, 453103, г. Стерлитамак, пр. Ленина, 37.
}

\begin{abstract}
Актуальность исследования связана с разработкой теоретических основ технологий подземного захоронения вырабатываемого промышленными объектами сероводорода с целью снижения его эмиссии в атмосферу. Рассматривается метод утилизации сероводорода в газогидратном состоянии, в котором одинаковое количество газа может храниться при значительно меньших значениях давления.

Целью исследования является выяснение особенностей протекания процесса образования газового гидрата $\mathrm{H}_{2} S$ при инжекции жидкого сероводорода в пористый резервуар.

объект: пористый пласт, насыщенный нефтью и водой, исходное давление которого ниже равновесного давления образования газогидрата сероводорода.

Методы исследования. На основе уравнений механики сплошной среды построена математическая модель тепломассопереноса в природном пласте, сопровождающегося образованием газогидрата сероводорода. Принято, что в рассматриваемом случае в пласте возникают три характерные зоны и соответственно два подвижных межфазных фронта - между первой и второй зонами, где происходит полный переход воды в газогидратное состояние (фронт гидратообразования), и между второй и третьей зонами, где осуществляется вытеснение нефти сероводородом (фронт вытеснения). Методом сведения к автомодельной переменной построены аналитические решения для безразмерных значений давления и температуры в каждой из трех областей пласта. Исследованы зависимости координаты и температуры межфазных границ от температуры закачиваемого сероводорода и исходной температуры пласта.

Результаты. Установлено, что с повышением начальной температуры пласта и температуры инжектируемого жидкого сероводорода координата фронта образования газогидрата $\mathrm{H}_{2} \mathrm{~S}$ уменьшается, а температура фронта вытеснения нефти сероводородом увеличивается. Показано, что при достаточно низких значениях температуры пласта и инжектируемого сероводорода может происходить выравнивание координат фронтов гидратообразования и вытеснения. На плоскости параметров «температура инжекции - начальная температура пласта» построены карты решений, т. е. критические диаграммы, определяющие существование режима, при котором фронт вытеснения нефти сероводородом опережает фронт образования газогидрата $\mathrm{H}_{2} \mathrm{~S}$.
\end{abstract}

\section{Ключевые слова:}

Математическая модель, автомодельное решение, пористая среда, фильтрация, газогидраты, сероводород.

\section{Введение}

Сероводород, принадлежащий к классу высокоопасных ядовитых загрязняющих веществ, является одним из основных отходов нефтехимических и нефтеперерабатывающих предприятий. В настоящее время сероводород относится к числу выбросов, вносящих наиболее существенный вклад в показатель «категория опасности предприятия», т. е. интегральный показатель, характеризующий изменения качества атмосферы через выбросы, учитывающий класс опасности вещества и оценивающий объем воздуха, необходимый для разбавления выбросов над территорией предприятия до уровня ПДК. Одним из методов снижения эмиссии в атмосферу сероводорода, вырабатываемого объектами промышленности, является его подземное захоронение в истощенных нефтяных коллекторах [1-4]. Поскольку при долгосрочном подземном хранении утилизируемых выбросов в виде флюида возникает риск их утечки на поверхность, некоторые авторы предлагают переводить их газогидратное состояние, в котором одинаковое количество газа может храниться при значительно меньших значениях давления [5-8].

Результаты экспериментального исследования образования газогидратов в пористых средах при- ведены, в частности, в работах [9-13]. В этих и других экспериментальных работах исследования образования газогидрата проводились в образцах небольшого размера. Поэтому в данных исследованиях процесс гидратообразования вследствие малых размеров образцов пористой среды лимитируется кинетическими механизмами (диффузией газа через пленки воды и газогидрата). Однако в случае природных протяженных пластов процесс гидратообразования лимитируется не кинетикой процесса, а фильтрацией и теплопереносом в пористой среде. Поэтому актуальным является построение математических моделей образования газогидратов в проницаемых пластах, учитывающих тепломассоперенос в пласте.

В настоящее время при численном моделировании процессов фильтрации и теплопереноса в пористом пласте, сопровождающихся образованием газогидрата, как правило, используются уравнения механики многофазных сред [14]. Математические модели гидратообразования в протяженных пористых пластах при закачке газа в пористую среду в исходном состоянии, насыщенную метаном и водой (льдом), сформулированы, в частности, в работах [15-19]. Математическая модель образования газогидрата $\mathrm{H}_{2} \mathrm{~S}$ в пласте, насы- 
щенном нефтью и водой, при инжекции жидкого сероводорода представлена в работе [20]. Однако в этой работе задача решена в наиболее простой постановке, когда образование газогидрата $\mathrm{H}_{2} \mathrm{~S}$ происходит на поверхности, совпадающей с поверхностью вытеснения нефти сероводородом. Поэтому в работе [20] предполагалось, что в пласте возникают только две характерные зоны и соответственно одна межфазная подвижная граница. В настоящей работе построена математическая модель образования газогидрата сероводорода для случая, когда гидратообразование происходит на фронтальной поверхности, которая не совпадает с поверхностью вытеснения нефти жидким сероводородом. Иными словами, рассматривается случай, когда в пласте возникают три характерные зоны и соответственно две межфазные подвижные границы.

\section{Постановка задачи}

Условия существования газового гидрата $\mathrm{H}_{2} \mathrm{~S}$ показаны на рис. 1 [7].



Рис. 1. Фазовая диаграмма системы «вода-сероводород»

Fig. 1. Phase diagram of the "water-hydrogen sulfide» system

На рисунке кривая $h g$ определяет равновесие между газообразным сероводородом, водой и газогидратом, кривая $h l$ - равновесие между жидким сероводородом, водой и газогидратом, а кривая $g l$ - двухфазное равновесие между газообразным и жидким сероводородом. Газогидрат $\mathrm{H}_{2} \mathrm{~S}$ стабильно существует в области, лежащей левее кривых $h g$ и $h l$. В квадрупольной точке $Q\left(p_{Q}=2,24 \mathrm{MПа} \mathrm{и}\right.$ $\left.T_{Q}=302,6 K\right)$ вода, газогидрат $\mathrm{H}_{2} \mathrm{~S}$, газообразный и жидкий сероводород находятся в равновесии.

Будем полагать, что поры полубесконечного пласта (который занимает полупространство $x>0$ ) в момент времени $t=0$ насыщены нефтью и водой. При этом исходная температура пласта $T_{0}$ выше температуры $T_{Q}$, соответствующей квадрупольной точке, т. е. исходное состояние пласта не соответствует условиям существования газогидрата $\mathrm{H}_{2} \mathrm{~S}$ (точка 0 на рис. 1). Пусть через левую границу пласта $(x=0)$ закачивается сероводород в жидком состоянии, температура $T_{e}$ и давление $p_{e}$ которого соответствуют условиям стабильного существования газогидрата $\mathrm{H}_{2} \mathrm{~S}$ (точка е на рис. 1).

В данной работе будем рассматривать случай, когда значение исходной водонасыщенности пласта не превышает 0,2 (т. е. воду можно считать неподвижной), а также модель с поршневым вытеснением нефти жидким сероводородом. Также будем рассматривать масштабы времени, значительно превышающие характерное время кинетики процесса образования гидрата $\mathrm{H}_{2} \mathrm{~S}$. Учитывая, что начальное состояние пласта не соответствует условиям существования газогидрата $\mathrm{H}_{2} \mathrm{~S}$, будем полагать, что гидратообразование происходит на фронтальной поверхности, которая не совпадает с поверхностью вытеснения нефти сероводородом. Следовательно, в рассматриваемом случае в пористом пласте образуются три характерные зоны. В первой (ближней) зоне поры насыщены сероводородом и газогидратом, во второй (промежуточной) зоне - сероводородом и водой, а в третьей (дальней) зоне - нефтью и водой. При этом образуются две подвижные межфазные поверхности: фронт гидратообразования (между первой и второй зонами) и фронт вытеснения нефти сероводородом (между второй и третьей зонами).

\section{Основные уравнения}

Система уравнений, описывающая процессы теплопереноса и фильтрации в проницаемом пласте, для рассматриваемой задачи запишется следующим образом [14-20]:

$$
\begin{gathered}
\frac{\partial}{\partial t}\left(\rho_{i} \phi S_{i}\right)=-\frac{\partial}{\partial x}\left(\rho_{i} u_{i}\right) \\
\rho c \frac{\partial T}{\partial t}=\frac{\partial}{\partial x}\left(\lambda \frac{\partial T}{\partial x}\right)-\rho_{i} c_{i} u_{i} \frac{\partial T}{\partial x} \\
u_{i}=-\frac{k_{i}}{\mu_{i}} \frac{\partial p}{\partial x} \\
k_{i}=k_{0} S_{i} \\
\rho_{i}=\rho_{0 i} \exp \left(\beta_{i}\left(p-p_{0}\right)\right) .
\end{gathered}
$$

Здесь $x$ - координата; $t$ - время; $p$ - давление; $T$ - температура; $\phi$ - пористость; нижние индексы $i=l, s$ относятся соответственно к параметрам нефти и жидкого сероводорода; $\rho_{i}-$ плотность; $k_{i}-$ фазовая проницаемость; $k_{0}$ - абсолютная проницаемость; $u_{i}$ - скорость фильтрации; $c_{i}-$ удельная теплоемкость; $\mu_{i}$ - динамическая вязкость; $\beta_{i}-$ коэффициент сжимаемости; $\mathrm{S}_{(i)}$ - насыщенность пор; $\lambda$ и $\rho c$ - эффективные значения коэффициента теплопроводности и объемной теплоемкости пласта. В дальнейшем $\lambda$ и $\rho c$ будем считать постоянными величинами, т. к. основной вклад в их значения вносят соответствующие параметры горной породы.

Поскольку на поверхности $x=x_{b}$, разделяющей первую и вторую зоны, происходит переход воды и сероводорода в газогидратное состояние, то из условий баланса тепла и массы сероводорода на этой поверхности следует: 


$$
\begin{gathered}
-\left(k_{s} \frac{\partial p}{\partial x}\right)_{b}^{-}+\left(k_{s} \frac{\partial p}{\partial x}\right)_{b}^{+}= \\
=\mu_{s} \phi S_{h}\left(\frac{\rho_{h} G}{\rho_{0 s}}+\frac{\rho_{h}(1-G)}{\rho_{w}}-1\right) V_{b}, \\
\lambda\left(\frac{\partial T}{\partial x}\right)_{b}^{-}-\lambda\left(\frac{\partial T}{\partial x}\right)_{b}^{+}=\phi \rho_{h} S_{h} L_{h} V_{b} .
\end{gathered}
$$

Здесь $V_{b}$ - скорость движения фронта образования газогидрата $\mathrm{H}_{2} \mathrm{~S} ; \rho_{w}$ - плотность воды; $\mathrm{S}_{w 0}-$ начальная водонасыщенность; $G$ - массовая концентрация сероводорода в гидрате; $L_{h}$ - удельная теплота образования газогидрата $\mathrm{H}_{2} \mathrm{~S}$. Нижний индекс $f$ относится к параметрам на поверхности, разделяющей первую и вторую зоны. Верхние индексы «-» и «+» относятся к значениям величин, терпящих разрыв на поверхности $x=x_{b}$, соответственно за фронтом образования гидрата $\mathrm{H}_{2} \mathrm{~S}$ (т. е. со стороны первой зоны) и перед данным фронтом (т. е. со стороны второй зоны). Температуру на этой поверхности будем полагать равной температуре квадрупольной точки $T_{Q}$.

Учитывая, что на данной поверхности выполняется также условие баланса массы воды:

$$
\phi \rho_{h} S_{h}(1-G) V_{b}=\phi \rho_{w} S_{w 0} V_{b} \text {, }
$$

для величины насыщенности пор газогидратом $\mathrm{H}_{2} \mathrm{~S}$ в первой зоне имеем:

$$
S_{h}=\frac{\rho_{w}}{\rho_{h}(1-G)} S_{w 0} .
$$

Поскольку на поверхности $x=x_{f}$, разделяющей вторую и третью области, происходит вытеснение нефти сероводородом, с учетом условий баланса массы нефти и сероводорода, а также баланса тепла на этой границе имеем:

$$
\begin{gathered}
-\left(k_{s} \frac{\partial p}{\partial x}\right)_{f}^{-}=\mu_{s} \phi\left(1-S_{w 0}\right) V_{f}, \\
-\left(k_{l} \frac{\partial p}{\partial x}\right)_{f}^{+}=\mu_{l} \phi\left(1-S_{w 0}\right) V_{f}, \\
\lambda\left(\frac{\partial T}{\partial x}\right)_{f}^{-}-\lambda\left(\frac{\partial T}{\partial x}\right)_{f}^{+}=0,
\end{gathered}
$$

где $V_{f}$ - скорость движения фронта вытеснения нефти сероводородом. Нижний индекс $f$ относится к параметрам на поверхности, разделяющей вторую и третью зону. Верхние индексы «-» и «十» относятся к значениям величин, терпящих разрыв на поверхности $x=x_{f}$, соответственно за фронтом вытеснения нефти сероводородом (т. е. со стороны второй зоны) и перед данным фронтом (т. е. со стороны третей зоны).

Поскольку значения коэффициентов сжимаемости жидкого сероводорода и нефти имеют порядок $10^{-9} \mathrm{\Pi a}^{-1}$, при перепадах давления в пласте порядка 1 МПа имеем: $\beta_{i}\left(p-p_{0}\right)<<1$, т. е. нефть и сероводород можно считать слабосжимаемыми жид- костями. Тогда, введя безразмерные параметры $P=p / p_{0}$ и $\theta=T / T_{0}$, на основе (1) получим:

$$
\begin{gathered}
\frac{\partial P_{j}}{\partial t}=\chi_{j}^{(p)} \frac{\partial}{\partial x}\left(\frac{\partial P_{j}}{\partial x}\right),(j=1,2,3), \\
\frac{\partial \theta_{j}}{\partial t}=\chi^{(T)} \frac{\partial}{\partial x}\left(\frac{\partial \theta_{j}}{\partial x}\right)+\gamma_{j} \chi^{(T)} \frac{\partial P_{j}}{\partial x} \frac{\partial \theta_{j}}{\partial x},(j=1,2,3),(4)
\end{gathered}
$$

где

$$
\begin{gathered}
\chi_{1}^{(p)}=\chi_{2}^{(p)}=\frac{k_{0}}{\phi \mu_{s} \beta_{s}}, \chi_{3}^{(p)}=\frac{k_{0}}{\phi \mu_{l} \beta_{l}}, \chi^{(I)}=\frac{\lambda}{\rho c}, \\
\gamma_{1}=\frac{k_{0}\left(1-S_{h}\right) \rho_{0 s} c_{s}}{\mu_{s} \beta_{s} p_{0} \lambda}, \gamma_{2}=\frac{k_{0}\left(1-S_{w 0}\right) \rho_{0 s} c_{s}}{\mu_{s} \beta_{s} p_{0} \lambda}, \\
\gamma_{3}=\frac{k_{0}\left(1-S_{w 0}\right) \rho_{01} c_{l}}{\mu_{l} \beta_{l} p_{0} \lambda} .
\end{gathered}
$$

Нижние индексы $j=1,2,3$ относятся к параметрам ближней, промежуточной и дальней зон соответственно.

\section{Автомодельное решение}

Введем автомодельную переменную $\xi=x / \sqrt{\chi^{(T)} t}$. Тогда уравнения (4) в каждой области примут вид:

$$
\begin{gathered}
-\xi \frac{d P_{j}}{d \xi}=2 \eta_{j} \frac{d}{d \xi}\left(\frac{d P_{j}}{d \xi}\right),(j=1,2,3), \\
-\xi \frac{d \theta_{j}}{d \xi}=2 \frac{d}{d \xi}\left(\frac{d \theta_{j}}{d \xi}\right)+2 \gamma_{j} \frac{d P_{j}}{d \xi} \frac{d \theta_{j}}{d \xi},(j=1,2,3),(5)
\end{gathered}
$$

где $\eta_{j}=\chi_{j}^{(p)} / \chi^{(T)}$.

После интегрирования (5) решения для безразмерных значений давления и температуры в каждой из зон могут быть записаны в виде:

$$
\begin{aligned}
& P_{1}=P_{b}+\left(P_{e}-P_{b}\right) \frac{\int_{\xi}^{\xi_{b}} \exp \left(-\frac{\xi^{2}}{4 \eta_{1}}\right) d \xi}{\int_{0}^{\xi_{b}} \exp \left(-\frac{\xi^{2}}{4 \eta_{1}}\right) d \xi}, \\
& \theta_{1}=\theta_{b}+\left(\theta_{e}-\theta_{b}\right) \frac{\int_{\xi}^{\xi} \exp \left(-\frac{\xi^{2}}{4}-2 \gamma_{1} P_{1}\right) d \xi}{\int_{0}^{\xi_{b}} \exp \left(-\frac{\xi^{2}}{4}-2 \gamma_{1} P_{1}\right) d \xi}, \\
& P_{2}=P_{f}+\left(P_{b}-P_{f}\right) \frac{\int_{\xi_{f}}^{\xi_{f}} \exp \left(-\frac{\xi^{2}}{4 \eta_{2}}\right) d \xi}{\int_{\xi_{b}}^{\xi_{f}} \exp \left(-\frac{\xi^{2}}{4 \eta_{2}}\right) d \xi}, \\
& \theta_{2}=\theta_{f}+\left(\theta_{b}-\theta_{f}\right) \frac{\int_{\xi}^{\xi_{f}} \exp \left(-\frac{\xi^{2}}{4}-2 \gamma_{2} P_{2}\right) d \xi}{\int_{\xi_{b}}^{\xi_{f}} \exp \left(-\frac{\xi^{2}}{4}-2 \gamma_{2} P_{2}\right) d \xi},
\end{aligned}
$$




$$
\begin{gathered}
P_{3}=1+\left(P_{f}-1\right) \frac{\int_{\xi}^{\infty} \exp \left(-\frac{\xi^{2}}{4 \eta_{3}}\right) d \xi}{\int_{\xi_{f}}^{\infty} \exp \left(-\frac{\xi^{2}}{4 \eta_{3}}\right) d \xi}, \\
\theta_{3}=1+\left(\theta_{f}-1\right) \frac{\int_{\xi}^{\infty} \exp \left(-\frac{\xi^{2}}{4}-2 \gamma_{3} P_{3}\right) d \xi}{\int_{\xi_{f}}^{\infty} \exp \left(-\frac{\xi^{2}}{4}-2 \gamma_{3} P_{3}\right) d \xi} .
\end{gathered}
$$

На основе соотношений (2) с учетом решений (6), (7) получим уравнения для определения координаты поверхности гидратообразования $\xi_{b}$ и значений параметров на нем:

$$
\begin{gathered}
k_{s 2} \frac{\left(P_{f}-P_{b}\right) \exp \left(-\frac{\xi_{b}^{2}}{4 \eta_{2}}\right)}{\int_{\xi_{b}}^{\xi_{f}} \exp \left(-\frac{\xi^{2}}{4 \eta_{2}}\right) d \xi}- \\
-k_{s 1} \frac{\left(P_{b}-P_{e}\right) \exp \left(-\frac{\xi_{b}^{2}}{4 \eta_{1}}\right)}{\int_{0}^{\xi_{b}} \exp \left(-\frac{\xi^{2}}{4 \eta_{1}}\right) d \xi}=A \xi_{b}, \\
\frac{\left(\theta_{b}-\theta_{e}\right) \exp \left(-\frac{\xi_{b}^{2}}{4}-2 \gamma_{1} P_{b}\right)}{\int_{0}^{\xi_{b}} \exp \left(-\frac{\xi^{2}}{4}-2 \gamma_{1} P_{1}\right) d \xi}- \\
-\frac{\left.\theta_{f}-\theta_{b}\right) \exp \left(-\frac{\xi_{b}^{2}}{4}-2 \gamma_{2} P_{b}\right)}{\int_{\xi_{b}}^{\xi_{f}} \exp \left(-\frac{\xi^{2}}{4}-2 \gamma_{2} P_{2}\right) d \xi}=B \xi_{b}, \\
\theta_{b}=T_{Q} / T_{0},
\end{gathered}
$$

где

$$
A=\phi \mu_{s} \chi^{(T)} S_{h}\left(\frac{\rho_{h} G}{\rho_{s 0}}+\frac{\rho_{h}(1-G)}{\rho_{w}}-1\right), B=\frac{\phi \rho_{h} L_{h} S_{h}}{2 \rho c} .
$$

Аналогично на основе соотношений (3) с учетом решений (7) и (8) получим систему уравнений для определения координаты поверхности вытеснения нефти сероводородом $\xi_{f}$ и значений параметров на нем:

$$
k_{s 2} \frac{\left(P_{b}-P_{f}\right) \exp \left(-\frac{\xi_{f}^{2}}{4 \eta_{2}}\right)}{\int_{\xi_{b}}^{\xi_{f}} \exp \left(-\frac{\xi^{2}}{4 \eta_{2}}\right) d \xi}=\phi \mu_{s} \chi^{(T)}\left(1-S_{w 0}\right) \xi_{f},
$$

$$
\begin{gathered}
k_{l} \frac{\left(P_{f}-1\right) \exp \left(-\frac{\xi_{f}^{2}}{4 \eta_{3}}\right)}{\int_{\xi_{f}}^{\infty} \exp \left(-\frac{\xi^{2}}{4 \eta_{3}}\right) d \xi}=\phi \mu_{l} \chi^{(T)}\left(1-S_{w 0}\right) \xi_{f}, \\
\frac{\left(\theta_{f}-\theta_{b}\right) \exp \left(-\frac{\xi_{f}^{2}}{4}-2 \gamma_{2} P_{f}\right)}{\int_{\xi_{b}}^{\xi_{f}} \exp \left(-\frac{\xi^{2}}{4}-2 \gamma_{2} P_{2}\right) d \xi}- \\
-\frac{\left(1-\theta_{f}\right) \exp \left(-\frac{\xi_{f}^{2}}{4}-2 \gamma_{3} P_{f}\right)}{\int_{\xi_{f}}^{\infty} \exp \left(-\frac{\xi^{2}}{4}-2 \gamma_{3} P_{3}\right) d \xi}=0 .
\end{gathered}
$$

Система граничных уравнений (9)-(14) в работе решена следующим образом. Вначале задается нулевое приближение искомых величин на фронте гидратообразования. Далее, решая уравнение (12), находим величину $P_{f}$ (как функцию $\xi_{f}$ ), подставляя которую в уравнение (13) получим трансцендентное уравнение для нахождения $\xi_{f}$. Решив данное уравнение (методом половинного деления), определим величину $\xi_{f}$ (и соответственно $P_{f}$ ), а затем из (14) определяем $\theta_{f}$. Далее, подставляя (11) в уравнение (10), получим трансцендентное уравнение для нахождения $\xi_{b}$. Решая данное уравнение (методом половинного деления), определяем новое приближение величины $\xi_{b}$. Затем, решая уравнение (9), находим новое приближение величины $P_{b}$. Циклически повторяя данную итерационную процедуру, получим последовательность значений, сходящуюся к искомым значениям параметров на межфазных границах.

Как было указано выше, в данной работе полагается, что вследствие рассматривания достаточно больших времен после начала инжекции сероводорода кинетикой процесса образования газогидрата $\mathrm{H}_{2} \mathrm{~S}$ можно пренебречь. Приведем оценки значений времени, при которых можно пренебречь переходной областью, в которой одновременно присутствуют сероводород, вода и газогидрат $\mathrm{H}_{2} \mathrm{~S}$, и толщина которой зависит от кинетики процесса образования газогидрата сероводорода.

Толщину вышеуказанной переходной области можно оценить следующим образом:

$$
\Delta x=\xi_{b}\left(\sqrt{\chi^{(T)}(t+\tau)}-\sqrt{\chi^{(T)} t}\right)
$$

где $\tau$ - характерное значение времени кинетики образования газогидрата $\mathrm{H}_{2} \mathrm{~S}$.

Поскольку протяженность первой зоны равна $x_{b}=\xi \sqrt{\tau^{(T)} t}$, а толщиной переходной области можно пренебречь, если $\Delta x<<x_{b}$, то имеем следующее условие: 


$$
\xi_{b}\left(\sqrt{\chi^{(T)}(t+\tau)}-\sqrt{\chi^{(T)} t}\right)<<\xi_{b} \sqrt{\chi^{(T)} t} .
$$

Откуда имеем: $t>>\tau / 3$.

Поскольку экспериментальное значение времени кинетики образования газогидрата $\tau$ в пористых средах с размерами зерен, характерных для природных пластов, в среднем составляет порядка нескольких часов, то рассматриваемая модель с четким фронтом образования газогидрата $\mathrm{H}_{2} \mathrm{~S}$ может применяться при масштабах времени после начала инжекции порядка нескольких суток.

\section{Результаты расчетов}

На рис. 2 приведены зависимости автомодельной координаты и температуры границ образования газогидрата $\mathrm{H}_{2} \mathrm{~S}$ (кривая 1) и вытеснения нефти сероводородом (кривая 2) от температуры закачиваемого сероводорода $(a)$ и начальной темпера-

\section{$a$}


туры пласта (b). Здесь и далее, если не оговорено иное для параметров, характеризующих систему, приняты следующие значения: $\phi=0,1, S_{w 0}=0,2$, $p_{0}=8 \mathrm{M \Pi а,} p_{e}=8,4 \mathrm{M \Pi а}, T_{0}=305 \mathrm{~K}, k_{0}=10^{-11} \mathrm{~m}^{2}$, $G=0,24, \lambda=2 \mathrm{BT} /(\mathrm{m} \cdot \mathrm{K}), \beta_{s}=3 \cdot 10^{-9} \Pi^{-1}, \beta_{l}=1 \cdot 10^{-9} \Pi^{-1}$, $\rho c=2 \cdot 10^{6}$ Дж/(К·кг), $L_{h}=4,1 \cdot 10^{5}$ Дж/кг, $\mu_{s}=2 \cdot 10^{-4} \Pi а \cdot c$, $\mu_{l}=2 \cdot 10^{-3} \Pi \mathrm{a} \cdot \mathrm{c}, \quad \rho_{h}=1003 \kappa \Gamma / \mathrm{M}^{3}, \quad \rho_{0 s}=890 \mathrm{\kappa} \Gamma / \mathrm{M}^{3}$, $\rho_{0 l}=900 \kappa \Gamma / \mathrm{M}^{3}, \rho_{w}=1000 \kappa \Gamma / \mathrm{m}^{3}, c_{s}=1800$ Дж/(К·кг), $c_{l}=1900$ Дж/(К·Кг). На рис. $2, a$ начальная температура пласта $T_{0}=305 \mathrm{~K}$, а на рис. $2, b$ температура закачиваемого сероводорода $T_{e}=290 \mathrm{~K}$.

Согласно рис. 2 , скорость второго фронта практически не зависит от температуры инжекции и начальной температуры пласта, а скорость первого фронта уменьшается с ростом этих параметров. Это обусловлено тем, что скорость фронта вытеснения нефти сероводородом лимитируется интенсивностью массопереноса в пласте, а скорость фронта ги-

$b$
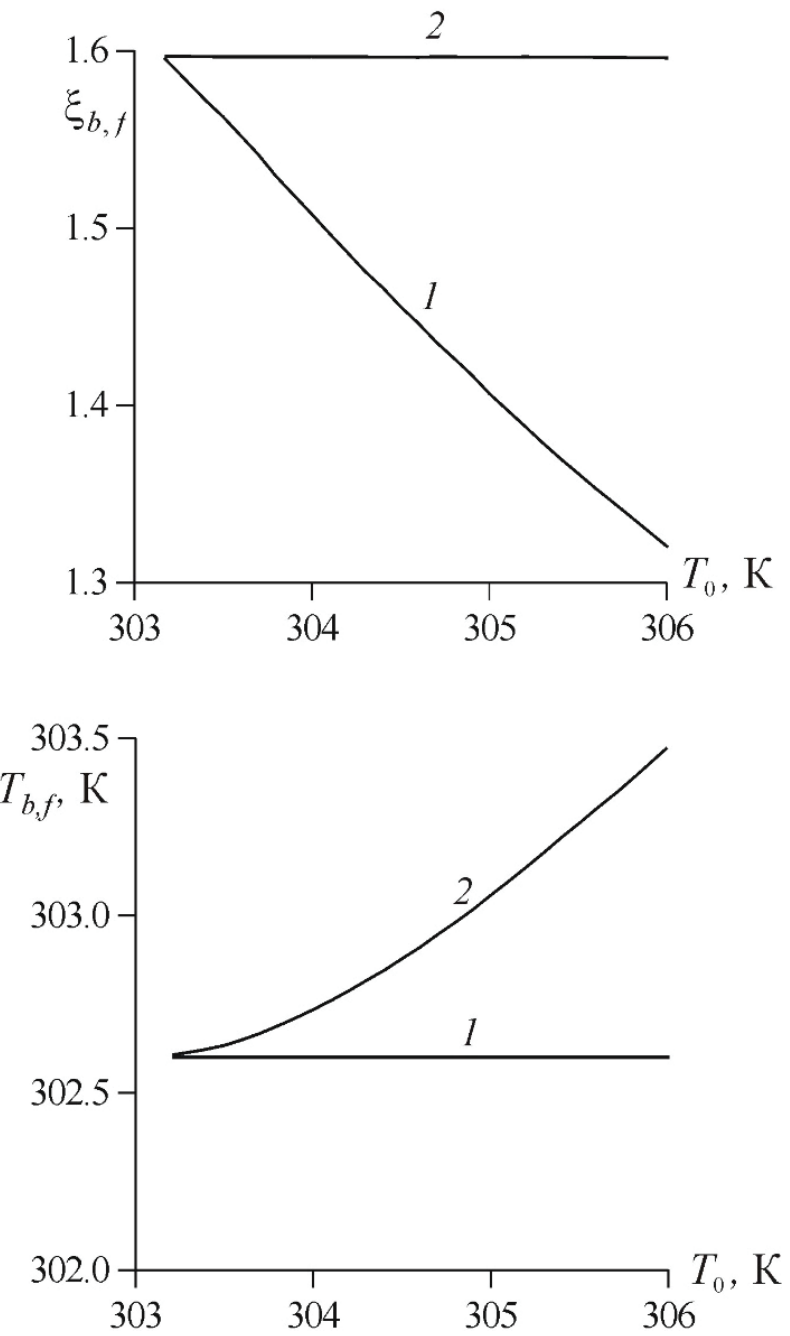

Рис. 2. Зависимость автомодельной координаты и температуры границ образования газогидрата Н. нефти сероводородом (кривая 2) от температуры закачиваемого сероводорода (a) и начальной температуры пласта (b)

Fig. 2. Dependence of the self-similar coordinate and temperature of the boundaries of $\mathrm{H}_{2} \mathrm{~S}$ gas hydrate (curve 1) formation and oil displacement by hydrogen sulfide (curve 2) on the temperature of the injected hydrogen sulfide (a) and the initial temperature of the layer (b) 
дратообразования лимитируется, прежде всего, отводом тепла, выделяющегося на первом фронте при гидратообразовании. Поэтому чем выше температура закачиваемого сероводорода и исходная температура пласта, тем ниже интенсивность отвода тепла, выделяющегося на границе гидратообразования.

Также рис. 2 показывает, что температура фронта вытеснения нефти сероводородом повышается с ростом температуры инжекции и начальной температуры пласта, а температура фронта образования гидрата $\mathrm{H}_{2} \mathrm{~S}$ остается постоянной. Это обусловлено тем, что положение первого фронта, согласно рис. 2 , не зависит от температуры инжекции и начальной температуры пласта, а температура на втором фронте равна температуре квадрупольной точки системы «вода-сероводород».

Также видно, что при достаточно низких значениях температуры инжекции и начальной температуры пласта координаты обоих фронтов выравниваются, т. е. скорость фронта гидратообразования возрастает до скорости фронта вытеснения нефти жидким сероводородом.

Таким образом, существует некоторое значение температуры закачиваемого сероводорода $T_{*}$ (назовем его критическим значением), выше которого реализуется рассматриваемый в данной работе режим с двумя различными межфазными границами. На рис. 3 приведены зависимости критического значения температуры инжектируемого сероводорода, соответствующего выравниванию координат обоих фронтов, от начальной температуры пласта при разных давлениях инжекции $p_{e}=8,3 \mathrm{MПа}$ (кривая 1) и 8,4 МПа (кривая 2). Согласно рис. 3, с увеличением начальной температуры пласта и давления инжекции сероводорода критическое значение температуры закачиваемого сероводорода снижается. Иными словами, режим с двумя различными межфазными границами реализуется при достаточно высоких значениях исходной температуры и давления инжекции. Это обусловлено тем, что с увеличением исходной температуры пласта снижается интенсивность отвода тепла, выделяющегося на границе гидратообразования, и соответственно уменьшается скорость фронта гидратообразования. А с увеличением давления инжекции возрастает скорость течения в пористой среде и соответственно увеличивается скорость фронта вытеснения нефти жидким сероводородом. Таким образом, при высоких значениях исходной

\section{СПИСОК ЛИТЕРАТУРЫ}

1. Machel H.G. Geological and hydrogeological evaluation of the Nisku Q-Pool in Alberta, Canada, for $\mathrm{H}_{2} \mathrm{~S}$ and/or $\mathrm{CO}_{2}$ storage // Oil Gas Sci. Technol. - 2005. - V. 60. - P. 51-65.

2. Numerical modeling of injection and mineral trapping of $\mathrm{CO}_{2}$ with $\mathrm{H}_{2} \mathrm{~S}$ and $\mathrm{SO}_{2}$ in a sandstone formation / T. Xu, J.A. Apps, K. Pruess, H. Yamamoto // Chemical Geology. 2007. - V. 24. № 3-4. - P. 319-346.

3. Factors affecting the chromatographic partitioning of $\mathrm{CO}_{2}$ and $\mathrm{H}_{2} \mathrm{~S}$ injected into a water-saturated porous medium / S. Bachu, температур пласта и давления инжекции сероводорода фронт вытеснения нефти сероводородом опережает фронт образования газогидрата $\mathrm{H}_{2} \mathrm{~S}$ даже при низких значениях температуры закачиваемого сероводорода.



Рис. 3. Зависимость критического значения температуры инжекции от начальной температуры пласта при $p_{e}=8,3$ МПа (кривая 1) и 8,4 МПа (кривая 2)

Fig. 3. Dependence of the critical injection temperature on the initial layer temperature at $p_{e}=8,3 \mathrm{MPa}$ (curve 1) and 8,4 MPa (curve 2)

\section{Заключение}

В результате математического моделирования инжекции жидкого сероводорода в пласт, насыщенный нефтью и водой, установлено, что с повышением начальной температуры пласта и температуры инжекции координата фронта образования газогидрата $\mathrm{H}_{2} \mathrm{~S}$ уменьшается, а температура фронта вытеснения нефти сероводородом увеличивается. Показано, что при низких значениях температуры инжекции и исходной температуры пористой среды режим с двумя межфазными поверхностями переходит в режим с одной межфазной поверхностью. Установлено, что критическое значение температуры закачиваемого сероводорода, выше которого реализуется режим с двумя различными межфазными границами, уменьшается с увеличением начальной температуры пласта.

Исследование выполнено при финансовой поддержке РФФИ и Республики Башкортостан (проект № 17-48-020123p_a).

D.B. Bennion, M. Pooladi-Darvish, H. Hong // Energy Procedia. - 2009. - V. 1. - P. 3165-3172.

4. Bachu S., Pooladi-Darvish M., Hong H. Chromatographic partitioning of impurities $\left(\mathrm{H}_{2} \mathrm{~S}\right)$ contained in a $\mathrm{CO}_{2}$ stream injected into a deep saline aquifer: P. 2. Effects of flow conditions // International Journal of Greenhouse Gas Control. - 2009. - V. 3. № 4. - P. 468-473.

5. Оценка возможности захоронения углекислого газа в криолитозоне Западной Сибири / А.Д. Дучков, Л.С. Соколова, Д.Е. Аюнов, М.Е. Пермяков // Криосфера Земли. - 2009. T. 13. - № 4. - C. 62-68. 
6. Чувилин Е.М., Гурьева 0.М. Экспериментальное изучение образования гидратов $\mathrm{CO}_{2}$ в поровом пространстве промерзающих и мерзлых пород // Криосфера Земли. - 2009. - Т. 13. № 3. - C. $70-79$.

7. Бык С.Ш., Макогон Ю.Ф., Фомина В.И. Газовые гидраты. М.: Химия, 1980. - 296 с.

8. Sloan E.D., Koh C.A. Clathrate Hydrates of Natural Gases. - Boca Raton: CRC Press, 2007. - 752 p.

9. Influence of Pore Size, Salinity and Gas Composition upon the Hydrate Formation Conditions / M. Yang, Y. Song, Y. Liu, Y. Chen, Q. Li // Chinese Journal of Chemical Engineering. 2010. - V. 18. - № 2. - P. 292-296.

10. Zhang P., Wu Q., Yang Y. Characteristics of Methane Hydrate Formation in Artificial and Natural Media // Energies. - 2013. V. 6. - № 3. - P. 1233-1249.

11. Livio R. Exploring methane-hydrate formation and dissociation in geologic materials through laboratory experiments: Kinetic behavior and morphology // Fuel. - 2015. - V. 141. - P. 173-184.

12. Preservation phenomena of methane hydrate in pore spaces / A. Hachikubo, S. Takeya, E. Chuvilin, V. Istomin // Physical Chemistry Chemical Physics. - 2011. - V. 13. - P. 17449-17452.

13. Чувилин Е.М., Гребенкин С.И. Экспериментальная оценка газопроницаемости газонасыщенных пород при гидратообразовании и замораживании // Криосфера земли. - 2015. - Т. 19. № 2. - C. 64-74.

14. Цыпкин Г.Г. Течения с фазовыми переходами в пористых средах. - М.: Физматлит, 2009. - 232 c.

\section{Информация об авторах}

Хасанов M.K., кандидат физико-математических наук, доцент кафедры прикладной информатики и программирования Стерлитамакского филиала Башкирского государственного университета.
15. Шагапов В.Ш., Рафикова Г.Р., Хасанов М.К. К теории образования газогидрата в частично водонасыщенной пористой среде при нагнетании метана // Теплофизика высоких температур. 2016. - T. 54. - № 6. - C. 911-920.

16. Гималтдинов И.К., Хасанов М.К. Математическая модель образования газогидрата при инжекции газа в пласт, частично насыщенный льдом // Прикладная математика и механика. 2016. - T. 80. - № 1. - C. 80-90.

17. Хасанов М.К. Численное моделирование закачки углекислого газа в истощенное месторождение углеводородов // Известия Томского политехнического университета. Инжиниринг георесурсов. - 2017. - Т. 328. - № 8. - С. 48-54.

18. Цыпкин Г.Г. Образование гидрата углекислого газа при его инжекции в истощенное месторождение углеводородов // Изв. РАН. Механика жидкости и газа. - 2014. - № 6. - С. 101-108.

19. Шагапов В.Ш., Чиглинцева А.С., Белова С.В. К теории процесса образования газогидрата в замкнутом теплоизолированном объеме, опрессованном метаном // Инженерно-физический журнал. - 2017. - Т. 90. - № 5. - С. 1208-1222.

20. Хасанов М.К. Инжекция жидкого сероводорода в пласт, насыщенный нефтью и водой // Вестник Тюменского государственного университета. Физико-математическое моделирование. Нефть, газ, энергетика. - 2017. - Т. 3. - № 2. - С. 72-84.

Поступила 13.03.2018 г. 
UDC 622.279.72

\title{
MATHEMATICAL MODEL OF HYDROGEN SULFIDE INJECTION INTO A RESERVOIR PARTIALLY SATURATED WITH WATER
}

\author{
Marat K. Khasanov, \\ hasanovmk@mail.ru \\ Sterlitamak Branch of Bashkir State University, \\ 37, Lenin Avenue, Sterlitamak, 453103, Russia.
}

The relevance of the research is related to the development of theoretical foundations of underground disposal technologies for hydrogen sulfide produced by industrial facilities for reducing its emission into the atmosphere. The paper considers the method of hydrogen sulfide utilization in the gas hydrate state, in which the same amount of gas can be stored at significantly lower pressures.

The aim of the research is to study the features of formation of $\mathrm{H}_{2} \mathrm{~S}$ gas hydrate when injecting liquid hydrogen sulfide into a porous reservoir.

Object of the research is a porous formation saturated with oil and water, the initial pressure of which is lower than the equilibrium pressure of formation of hydrogen sulfide gas hydrate.

Methods. B Based on the equations of continuum mechanics the authors have developed the mathematical model of heat and mass transfer in a natural reservoir, accompanied by formation of hydrogen sulfide gas hydrate. It is assumed that in the case under consideration, three characteristic zones and, respectively, two moving interphase front arise in the reservoir: between the first and the second zones where the water completely passes to the gas hydrate state (the hydrate formation front) and between the second and the third zones where oil is displaced by hydrogen sulfide (displacement front). Using the method of reduction to the self-similar variable the authors constructed the analytical solutions for the dimensionless pressure and temperature values in each of the three regions of the reservoir and studied the dependence of the coordinate and temperature of the interface boundaries on the temperature of the injected hydrogen sulfide and the initial temperature of the formation.

Results. It was established that the coordinate of the formation front of $\mathrm{H}_{2} \mathrm{~S}$ gas hydrate decreases, and the temperature of the front of oil displacement by hydrogen sulfide increases when the initial temperature of the formation and temperature of injected liquid hydrogen sulfide grow. It is shown that at sufficiently low values of reservoir temperature and injected hydrogen sulfide, the coordinates of hydrate formation and displacement fronts can be aligned. The authors constructed the solution maps in the parameter plane «injection temperature - the initial temperature of the reservoirn, i. e. critical diagrams that determine the existence of a mode in which the front of oil displacement by hydrogen sulfide is ahead of formation front of $\mathrm{H}_{2} \mathrm{~S}$ gas hydrate.

\section{Key words}

Mathematical model, self-similar solution, porous medium, filtration, gas hydrates, hydrogen sulfide.

This research was supported by the Russian Foundation for Basic Research and the Republic of Bashkortostan (project no. 17-48-020123).

\section{REFERENCES}

1. Machel H.G. Geological and hydrogeological evaluation of the Nisku Q-Pool in Alberta, Canada, for $\mathrm{H}_{2} \mathrm{~S}$ and/or $\mathrm{CO}_{2}$ storage. Oil Gas Sci. Technol., 2005, vol. 60, pp. 51-65.

2. Xu T., Apps J. A., Pruess K., Yamamoto H. Numerical modeling of injection and mineral trapping of $\mathrm{CO}_{2}$ with $\mathrm{H}_{2} \mathrm{~S}$ and $\mathrm{SO}_{2}$ in a sandstone formation. Chemical Geology, 2007, vol. 24, no. 3-4, pp. 319-346.

3. Bachu S., Bennion D.B., Pooladi-Darvish M., Hong H. Factors affecting the chromatographic partitioning of $\mathrm{CO}_{2}$ and $\mathrm{H}_{2} \mathrm{~S}$ injected into a water-saturated porous medium. Energy Procedia, 2009, vol. 1, pp. 3165-3172.

4. Bachu S., Pooladi-Darvish M., Hong H. Chromatographic partitioning of impurities $\left(\mathrm{H}_{2} \mathrm{~S}\right)$ contained in a $\mathrm{CO}_{2}$ stream injected into a deep saline aquifer: P. 2. Effects of flow conditions. International Journal of Greenhouse Gas Control, 2009, vol. 3, no. 4, pp. $468-473$.

5. Duchkov A.D., Sokolova L.S., Ayunov D.E., Permyakov M.E. Assessment of potential of west Siberian permafrost for the carbon dioxide storage. Earth's Cryosphere, 2009, vol. 13, no. 4, pp. $62-68$.

6. Chuvilin E.M., Guryeva 0.M. Experimental investigation of $\mathrm{CO}_{2}$ gas hydrate formation in porous media of frozen and freezing sediments. Earth's Cryosphere, 2009, vol. 13, no. 3. pp. 70-79.

7. Byk S.Sh., Makogon Yu.F., Fomina V.I. Gazovye gidraty [Gas hydrates]. Moscow, Khimiya Publ., 1980. 296 p.
8. Sloan E.D., Koh C.A. Clathrate Hydrates of Natural Gases. Boca Raton, CRC Press, 2007. 752 p.

9. Yang M., Song Y., Liu Y., Chen Y., Li Q. Influence of Pore Size, Salinity and Gas Composition upon the Hydrate Formation Conditions. Chinese Journal of Chemical Engineering, 2010, vol. 18, no. 2, pp. 292-296.

10. Zhang P., Wu Q., Yang Y. Characteristics of Methane Hydrate Formation in Artificial and Natural Media. Energies, 2013, vol. 6, no. 3, pp. 1233-1249.

11. Livio R. Exploring methane-hydrate formation and dissociation in geologic materials through laboratory experiments: Kinetic behavior and morphology. Fuel, 2015, vol. 141, pp. 173-184.

12. Hachikubo A., Takeya S., Chuvilin E., Istomin V. Preservation phenomena of methane hydrate in pore spaces. Physical Chemistry Chemical Physics, 2011, vol. 13, pp. 17449-17452.

13. Chuvilin E.M., Grebenkin S.I. Gas permeability variations in gasfilled soils upon hydrate formation and freezing: An experimental study. Earth's Cryosphere, 2005, vol. 19, no. 2. pp. 59-64.

14. Tsypkin G.G. Techeniya s fazovymi perekhodami v poristykh sredakh [Flows with phase transitions in porous media]. Moscow, Fizmatlit Publ., 2009. 232 p.

15. Shagapov V.Sh., Rafikova G.R., Khasanov M.K. On the Theory of Formation of Gas Hydrate in Partially Water-Saturated Porous Medium when Injecting Methane. High Temperature, 2016, vol. 54, no. 6, pp. 858-866. In Rus.

16. Gimaltdinov I.K., Khasanov M.K. Mathematical model of the formation of a gas hydrate on the injection of gas into a stratum par- 
tially saturated with ice. Journal of Applied Mathematics and Mechanics, 2016, vol. 80, no. 1, pp. 57-64. In Rus.

17. Khasanov M.K. Numerical simulation of carbon dioxide injection into a depleted hydrocarbon deposit. Bulletin of the Tomsk Polytechnic University. Geo Assets Engineering, 2017, vol. 328, no. 8, pp. 48-54. In Rus.

18. Tsypkin G.G. Formation of carbon dioxide hydrate at the injection of carbon dioxide into a depleted hydrocarbon field. Fluid $D y$ namics, 2014, vol. 49, no. 6, pp. 789-795. In Rus.
19. Shagapov V.Sh., Chiglintseva A.S., Belova S.V. On the Theory of Formation of a Gas Hydrate in a Heat-Insulated Space Compacted with Methane. Journal of Engineering Physics and Thermophysics, 2017, vol. 90, no. 5, pp. 1147-1161. In Rus.

20. Khasanov M.K. Injection of liquid hydrogen sulfide in a layer saturated with oil and water. Tyumen State University Herald. Physical and Mathematical Modeling. Oil, Gas, Energy, 2017, vol. 3, no. 2, pp. 72-84. In Rus.

Received: 13 March 2018.

\section{Information about the authors}

Marat K. Khasanov, Cand. Sc., associate professor, Sterlitamak Branch of Bashkir State University. 